\title{
Laser-induced fluorescence saturation effects on ion velocity distribution functions in the vicinity of reflecting surfaces
}

\author{
V. Pigeon, ${ }^{\text {a) }}$ (D) N. Claire, C. Arnas, and F. Doveil \\ AFFILIATIONS \\ Aix-Marseille Univ, CNRS, PIIM 13013 Marseille, France \\ ${ }^{a)}$ Electronic mail: valentin.pigeon@univ-amu.fr
}

\begin{abstract}
Laser-induced fluorescence (LIF) measurements of Argon ions performed in the sheath/pre-sheath facing a floating metallic plate and a $\mathrm{BNSiO}_{2}$ ceramic one immersed in a low temperature plasma exhibit unexpected features. It appears that a strong fluorescence signal which could be unduly attributed to ions moving backward in the sheath is detected, even though the floating potential is far below the plasma potential. Moreover, this signal may be stronger than the one corresponding to ions having a forward motion. It is demonstrated that this abnormal measurement is due to the optical pumping saturation of the incident laser beam, while this saturation does not exist for the scattering of the beam at the sample surface. The reflected signal is unambiguously identified using a theoretical beam scattering model. It is also shown that the presence of the sheath/presheath density gradient is able to trigger the LIF saturation effect, complicating ion density measurements.
\end{abstract}

\section{INTRODUCTION}

Laser-Induced Fluorescence (LIF) diagnostic has been used in plasmas for decades. ${ }^{1-3}$ This diagnostic relies on the excitation of an electronic transition of atoms or ions. Using a tunable laser, it is possible to combine it with the Doppler shift effect to obtain the atomic or ionic velocity distribution function (IVDF) along the laser beam. Generally, as for the Argon ionic scheme used in the following experiments, shown in Fig. 1 , atoms or ions are excited from level 1 , usually a metastable one to have a sufficient lifetime to represent ion behavior, to an excited low lifetime level 2. The fluorescence signal corresponds to the emitted photon from the level 2 to level 3 transitions. The main advantage of this technique is its noninvasive nature which is highly relevant for plasmas that are known to be easily perturbed.

During the past few decades, several LIF extensions have been developed. Laser-collision induced fluorescence ${ }^{4}$ (LCIF) allows the determination of electron density and temperature thanks to the excitation of electronic states populated by laser excitation and electron collisions; planar LIF $^{5}$ (PLIF) uses a laser sheet beam to make $2 \mathrm{D}$ resolved measurements; $3 \mathrm{D} \mathrm{LIF}^{6}$ extends the PLIF to the third dimension; laser-induced fluorescence-dip ${ }^{7}$ (LIF-dip) allows the measurement of the local electric field thanks to the excitation of Rydberg states which are sensitive to the Stark effect; velocity-space cross-correlation $^{8}$ allows detection of plasma fluctuations; optical tagging 9 and nonlinear optical tagging ${ }^{10}$ are used to measure transport in the velocity space.

While being well developed and mastered, some experimental artifacts exist and have to be cautiously considered like laser beam absorption, ${ }^{11}$ spatially inhomogeneous laser intensity ${ }^{12}$ Stark shift, ${ }^{13}$ ion metastable cross section dependence on electron temperature, ${ }^{14}$ and optical pumping saturation. ${ }^{15-18}$

The LIF diagnostic is well fitted for plasma sheath investigation. It has been successfully applied to sheaths facing metal samples ${ }^{11,19}$ and gave quantitatively good agreements with theory and simulations. ${ }^{20,21}$ Some experiments have been performed in plasma sheaths with a wide variety of purposes. ${ }^{22-2}$

Moreover, performing LIF in a sheath implies that a surface is present and reflects the laser beam, which may cause some undesired additional signals. This can be avoided by drilling a hole on the surface ${ }^{25,29}$ and/or using a beam dump to cancel the beam reflection out. ${ }^{25,29,30}$ Also, the experimental set-up has to provide a resolution high enough to probe this plasma region 


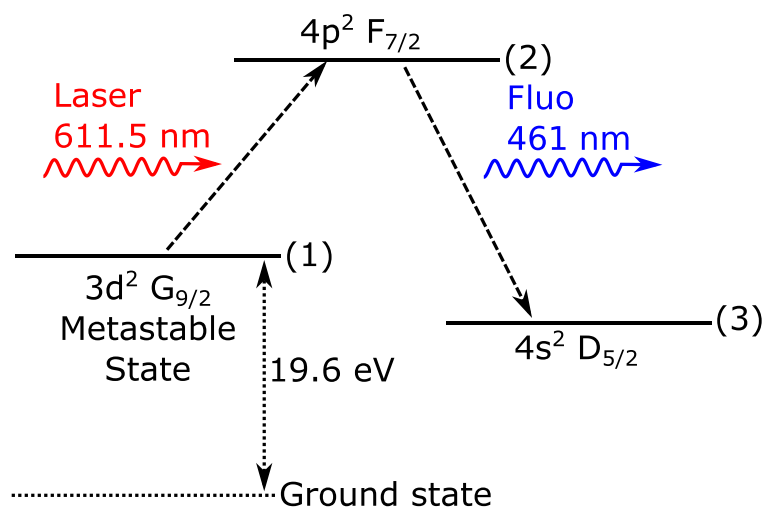

FIG. 1. Basic 1-photon Argon ion LIF scheme. The metastable state is chosen for his long lifetime, and his relative abundance among the ions. The example shown here is the one used in the following experiments.

where the electric potential gradient and the induced spatial gradient of the IVDF are steep.

A well-known bias of the LIF diagnostics is the saturation of the used optically pumped electronic transition. ${ }^{15,17}$ This happens when the power density of the laser is so high that the optical pumping rate of atoms or ions from atomic levels 1 to 2 is on the order of magnitude or larger than the collision creation rate of the first level ions or atoms. In this regime, the signal intensity is no more proportional to the laser power density as no more ions/atoms may be excited. ${ }^{18}$

High laser power density also leads to a broadening of the measured velocity distribution function, especially when the spectral width of the laser is close to the transition's one. Indeed, the laser emission ray is, in simple cases, a gaussian function with a finite spectral width. When the power density is too high, the photons located at the peak of the gaussian saturate the LIF signal, while those located on the wings do not and then excite ions with higher/lower velocities, eventually broadening the IVDF. ${ }^{17}$ This effect can be avoided either by working with low laser power or by using a deconvolution technique which extracts the real distribution function from the experimental signal knowing the broadening function and considering it as constant with respect to the observed phenomenon. ${ }^{28,31}$

The present article discusses LIF diagnostic saturation artifacts in plasma sheaths/pre-sheaths occurring when the saturation effects are combined with laser beam reflection on the surface. ${ }^{32}$ It is shown that, when the laser power density is high enough to trigger the optical pumping saturation, the signal coming from the reflected beam may become larger than the one coming from the incident beam. This misleading effect has been studied in a low temperature plasma discharge in front of a metallic unbiased plate and a ceramic one, both with a floating potential far below plasma potential. The influence of the density gradient in the sheath on the LIF data treatment and interpretation is also discussed.

Also, the effect of the laser scattering on the measured IVDF shape is discussed and is shown to be useful to discriminate the laser reflection from the incident beam signal in the measured distribution functions.
This paper is organized as follows: Section II presents the experimental set-up. Section III defines laser saturation effects in the bulk plasma. Section IV describes saturation effects in the plasma sheath in front of a conducting plate and a ceramic plate. The effect of the scattering on the measured IVDF shape is discussed in Sec. V. The conclusions are presented in Sec. VI.

\section{EXPERIMENTAL SET-UP}

The experiments are performed in a multipolar plasma device shown in Fig. 2 and already described in Refs. 19 and 33. The discharge is created by two Tungsten filaments negatively biased to $100 \mathrm{~V}$ with respect to the grounded vacuum vessel. The heating current is monitored in order to maintain a $0.5 \mathrm{~A}$ stable discharge current. A turbomolecular pump provides a base pressure of a few $10^{-6}$ mbar and the working pressure is $10^{-4} \mathrm{mbar}$ of Argon gas. Permanent magnets placed around the vacuum chamber in a staggered pattern allow the confinement of the ionizing electrons. These ionizing electrons may experience different confinement regimes depending on the neutral gas pressure and the discharge current. ${ }^{34}$ With the used discharge parameters, the electrons are well described by a bi-maxwellian distribution function. ${ }^{25}$ The cold plasma electrons have a temperature $\mathrm{T}_{e} \simeq 1.5 \mathrm{eV}$ and a density $n_{e} \simeq 10^{15} \mathrm{~m}^{-3}$. The hot ionizing electrons temperature is $\mathrm{T}_{e h} \simeq 12 \mathrm{eV}$ and their relative concentration is $\frac{n_{e h}}{n_{e h}+n_{e}} \simeq 15 \%$, where $n_{e h}$ is the hot electron density. These parameters are deduced from a non-linear fit of a Langmuir probe's I-V characteristic.

The LIF is operated thanks to a Coherent 899 tunable 250 $\mathrm{mW}$ dye laser with an $8 \mathrm{~mm}$ diameter beam pumped by a Coherent Verdi solid state laser. The LIF scheme uses the $3 \mathrm{~d}^{2} \mathrm{G}_{9 / 2}$ metastable Argon ion state, optically pumped to the $4 \mathrm{p}^{2} \mathrm{~F}_{7 / 2}$ state at $611.5 \mathrm{~nm}$, as shown in Fig. 1. The fluorescence signal corresponds to the $4 \mathrm{p}^{2} \mathrm{~F}_{7 / 2}$ to $4 \mathrm{~s}^{2} \mathrm{G}_{9 / 2}$ transition, leading to a fluorescence signal at $461 \mathrm{~nm}$ detected by a photomultiplier tube (PMT). A slit and a lens assembly fix the spatial resolution of the diagnostics which cannot be lower than $0.1 \mathrm{~mm}$. The used resolution is $1 \mathrm{~mm}$. The used measurement technique is photon counting: photomultiplier fronts are counted on a given amount of time ( $2 \mathrm{~ms})$, for laser on and laser off. This is repeated 200 times and summed, and then, the laser off counts are subtracted to the laser on counts, eventually giving the fluorescence signal. This is done for 50 frequencies in a $15 \mathrm{GHz}$ range, which provides the complete IVDF. The time sampling and repetitions can be increased to improve the signal to noise ratio; with these parameters, the typical number of counts for the IVDF maximum is $10^{4}$. The photon counting technique also allows IVDF time resolved measurements; however, it has not been used here.

Measurements are performed in front of two disks: a $4 \mathrm{~cm}$ diameter floating stainless steel plate and a $2 \mathrm{~cm}$ diameter ceramic plate $\left(\mathrm{BNSiO}_{2}\right)$ located at the center of the device. The discharge parameters set the Debye length around $0.2 \mathrm{~mm}$, leading to a few millimeters large sheath. The charge exchange mean free path is a few tens of centimeters long, giving the typical scale of the pre-sheath (the electron ionization mean free path is of the order of 1 meter). The Bohm velocity is close to $1.9 \mathrm{~km} / \mathrm{s}$. 


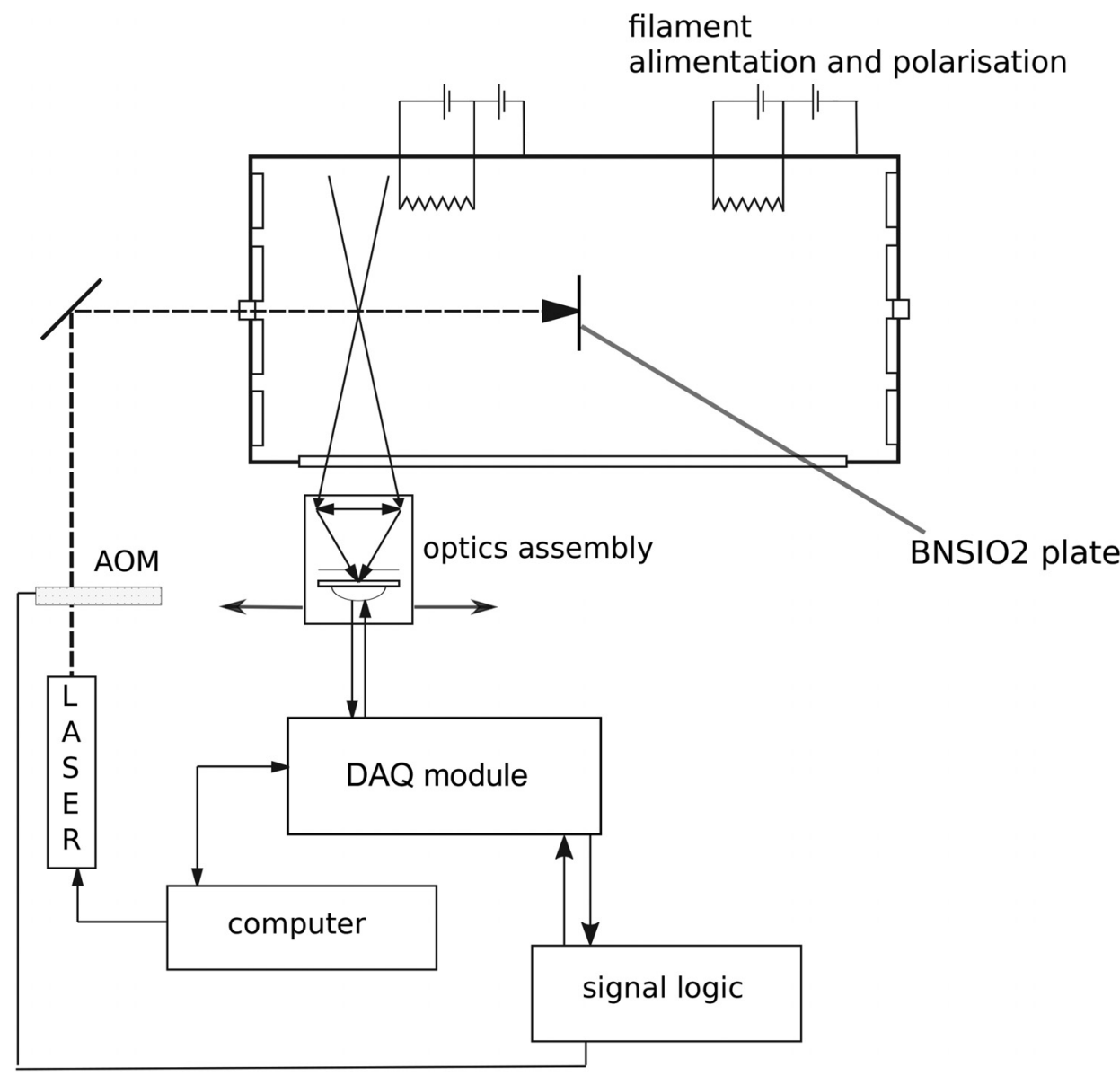

FIG. 2. Sketch of the multipolar device. The DAQ module driven by the computer controls the laser scan and the photomultiplier tube displacement. The acousto-optic modulator (AOM) switches the laser on and off, in order to identify the LIF signal among the spontaneous light emitted by the plasma.

\section{EFFECT OF THE SATURATION ON THE BULK PLASMA}

Several IVDFs have been recorded far enough from the floating conducting plate $(20 \mathrm{~cm})$ in order to measure a single nonshifted maxwellian distribution function. ${ }^{19}$ These distributions, and the following ones nearby the surface, are measured while varying the laser power thanks to different optical filters. Then,

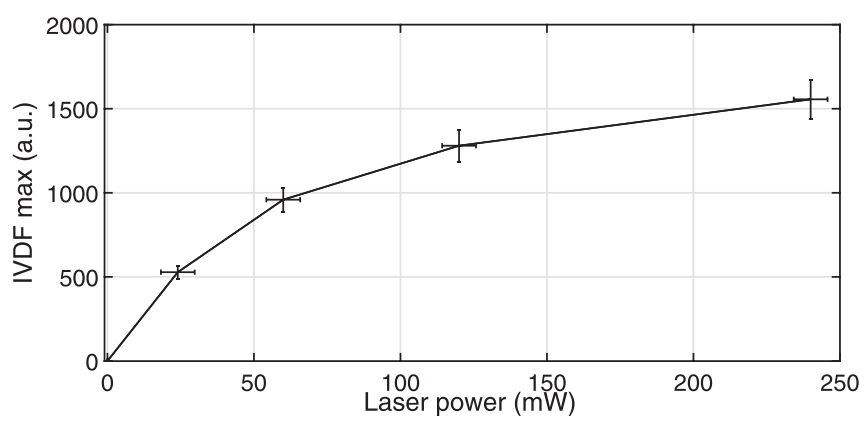

FIG. 3. IVDF maximum vs laser beam power, measured $20 \mathrm{~cm}$ away from the wall where the distribution function is maxwellian. the maximum signal of the IVDFs, the density $n=\int f(v) d v$, the fluid velocity $\mathrm{V}=\frac{\int v f(v) d v}{n}$, and the temperature $\mathrm{T}=m_{i} \frac{\int(\mathrm{V}-v)^{2} f(v) d v}{n}$ can be computed.

The maximum IVDF intensity in the bulk plasma with respect to the laser power is shown in Fig. 3. IDVF measurements below $24 \mathrm{~mW}$ were possible, but the signal-to-noise ratio was too low. Thereby, they were not accounted for. This plot demonstrates that the LIF signal saturates, and according to these maximum values, it is considered that the laser power threshold for LIF saturation to occur is below $60 \mathrm{~mW}$ and that measurements at $24 \mathrm{~mW}$ are unsaturated. No significant increase in the temperature with the laser power was observed.

\section{EFFECT OF THE SATURATION ALONG THE SHEATH}

IVDFs have been measured in the vicinity of the stainless steel and ceramic plates, at different locations. For both samples, the IVDF exhibits two peaks: the positive velocities peak named $\mathrm{P}^{+}$, and the negative velocities one $\mathrm{P}^{-}$. The presence of two peaks is due to the reflection of the laser beam on the plate, which excites the drifting ions twice. Since the floating potential is $\phi_{f}=-40 \mathrm{~V}$ for the metal plate, the ions are necessarily 


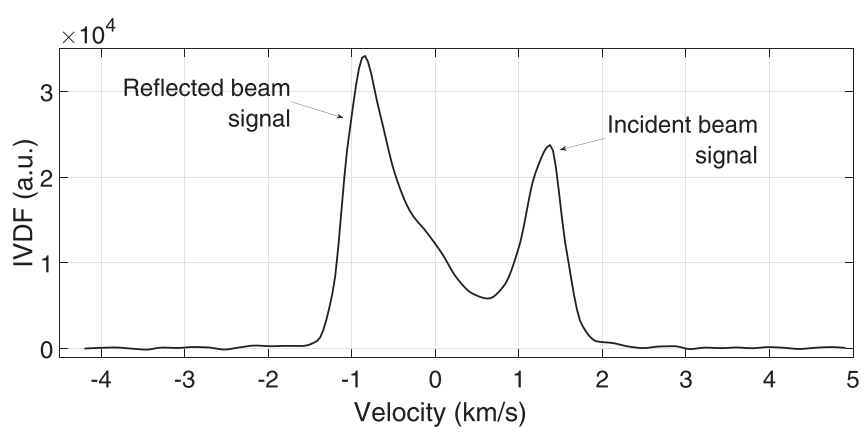

FIG. 4. IVDF showing that the reflected laser beam gives a larger signal intensity than the incident beam. Measured $8 \mathrm{~mm}$ away from the ceramic sample.

accelerated toward the sample and the corresponding signal is $\mathrm{P}^{+}$. Therefore, $\mathrm{P}^{-}$corresponds to the reflected beam.

On the other hand, for the ceramic plate, the floating potential is a priori unknown and the IVDF could show unexpected characteristics. Indeed in Fig. $4, \mathrm{P}^{-}$is larger than $\mathrm{P}^{+}$, suggesting that more ions flow toward the plasma than toward the wall, which could imply, at first glance, the presence of a Space Charge Limited (SCL)/inverse sheath. ${ }^{35,36}$ These peculiar forms of sheath occur when the electron emission of the surface is strong and are characterized by a potential well in front of the wall and a positive floating wall potential, respectively. Ceramics are known to be stronger emitters than metals, so this hypothesis cannot be excluded. These two structures could induce a backward motion of the ions; however, this has not yet been experimentally observed.

\section{A. Influence of the laser power on the IVDF}

It appears that the ratio between $\mathrm{P}^{-}$and $\mathrm{P}^{+}$heights depends on the laser power. This is visible in Fig. 5 which shows the ratio between $\mathrm{P}^{+}$and $\mathrm{P}^{-}$maxima. The higher the power, the lower the ratio for locations far from the surface. It can be seen that this effect also occurs for the metal plate. Moreover, the inversion of the ratio occurs below $60 \mathrm{~mW}$, suggesting that this effect is related to the saturation of the LIF that was highlighted in Sec. III.

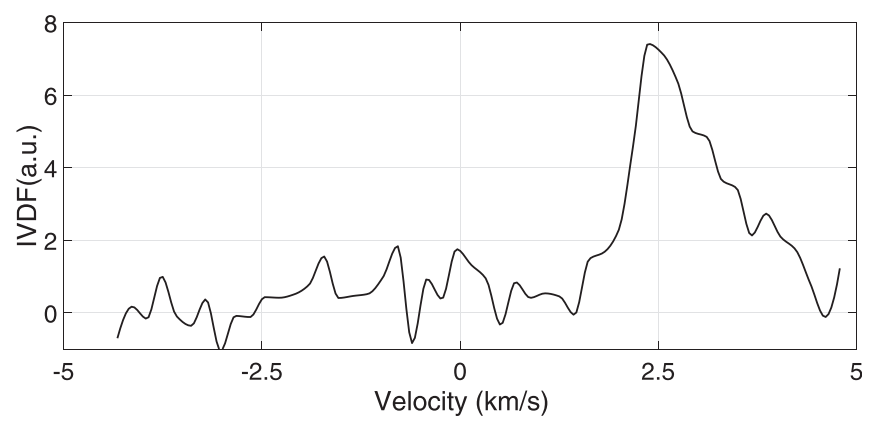

FIG. 6. IVDF measured at $8 \mathrm{~mm}$, with the laser propagating through a $0.8 \mathrm{~mm}$ hole drilled inside the ceramic sample. $P^{-}$vanishes.

To understand this phenomenon, a $0.8 \mathrm{~mm}$ hole was firstly drilled in the ceramic plate in order to propagate the laser through it. To achieve this, the laser was focused to reduce its diameter. As a result the laser reflection was discarded, allowing the determination of the signal coming from the incident beam. The result is shown in Fig. 6 where only forward moving ions are detected. Therefore, it is deduced that $\mathrm{P}^{-}$corresponds to the reflected beam. Considering that the drilled hole diameter is small enough so that the Debye screening is effective and neglecting other possible surface effects induced by the hole, it can be considered that the measured ions only flow toward the surface and the sheath potential determination is possible from the measurement of the ion mean velocity.

The fact that $\mathrm{P}^{-}$may become larger than $\mathrm{P}^{+}$is due to the combination of the LIF saturation effect, the geometry of the collecting optics, and the scattering of the laser beam on the surface. First, at laser powers higher than $24 \mathrm{~mW}$, it has been shown that the incident beam saturates the transition, as the signal intensity does not depend linearly on the laser power density (Fig. 3): the $\mathrm{P}^{+}$intensity slightly varies with power. On the other hand, the reflected beam has a lower power density, which decreases with the wall distance. Indeed, the reflection at the ceramic surface is diffuse due to the porosity of the $\mathrm{BNSiO}_{2}$ surface. The resulting power density allows the reflected beam not to saturate the transition: as shown in Fig. 7, the $\mathrm{P}^{-}$intensity linearly varies with the laser power. Moreover, the space

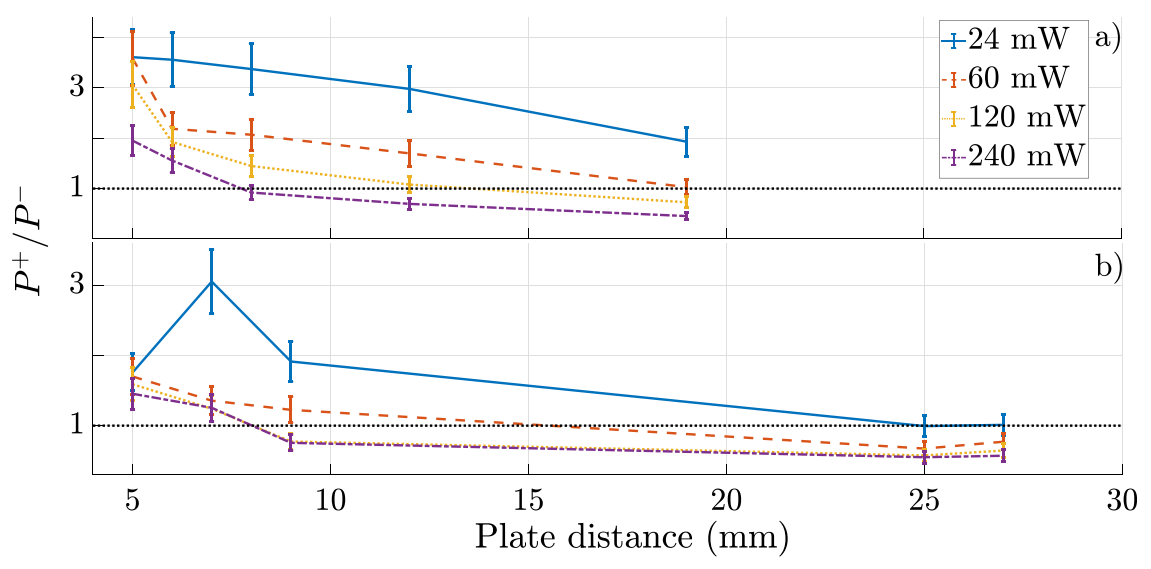

FIG. 5. Ratio between incident and reflected beam peak maxima for (a) the ceramic plate and (b) the steel plate. The ratio is larger than 1 for low laser power and smaller than 1 for high laser powers and evolves along the pre-sheath, increasing as the plate distance decreases. The $\frac{P^{+}}{P^{-}}=1$ ratio is represented by the horizontal black dotted line. 


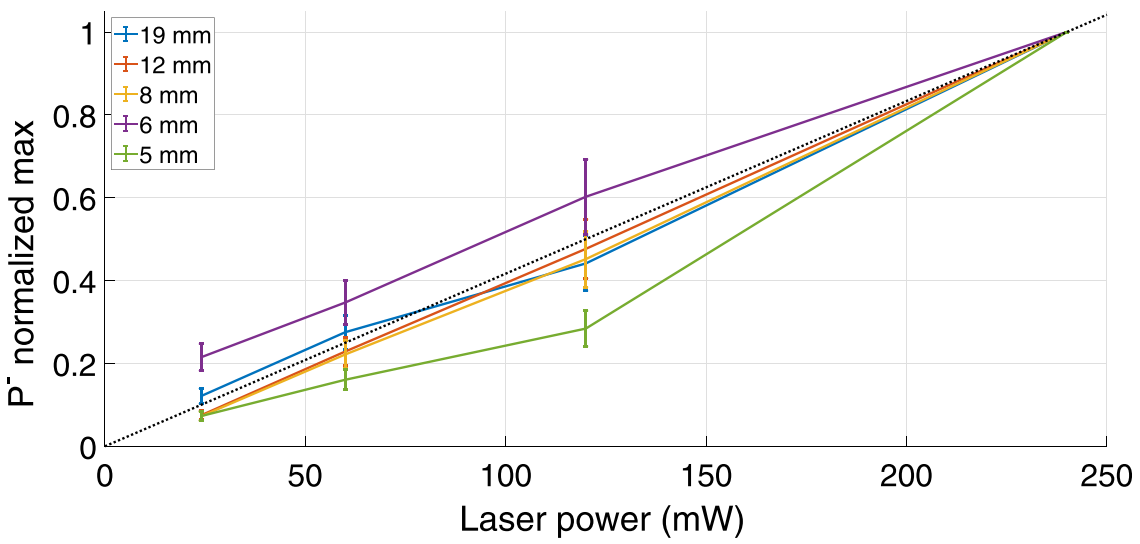

FIG. 7. $P^{-}$normalized maximum intensity signal as a function of laser power for the ceramic plate. The intensity at each location is normalized with respect to its value at $240 \mathrm{~mW}$. The black dotted line represents the linear variation, which is the one expected in the non-saturation regime.

occupied by the reflected beam in the photon collecting volume defined by the collecting optics can increase with the surface distance. Therefore, the reflected beam may generate more signals away from the sample. At high laser power for which $\mathrm{P}^{+}$saturates, this effect also contributes to the $\frac{\mathrm{P}^{+}}{\mathrm{P}^{-}}$ratio increase shown in Fig. 5 as one gets closer to the plate. A decrease is observed when the scattering of the beam is lower.

The reason why this effect is also present for the metal plate, while being weaker, might be due to an unpolished surface corresponding to a rough oxide layer. When the reflection tends toward specular reflection, the difference between the reflected and incident beam power density decreases and the reflected beam may also saturate the transition, except at the location where the density is high and the incident beam does not saturate.

Finally, the decrease in the slit width led to the $\mathrm{P}^{+}$intensity signal higher than the $\mathrm{P}^{-}$one, also suggesting that the size of the photon detection volume combined with the diffuse reflection plays a major role in this effect. Let us recall that the resolution used in all the experiments discussed here was $1 \mathrm{~mm}$, and was reduced to $0.7 \mathrm{~mm}$ to test this.

This feature may lead to biased interpretation of the data, if it is assumed that the largest peak of the IVDF always corresponds to the incident laser beam. ${ }^{31}$ Also, if the two peaks have similar intensities and the floating potential of the considered surface is unknown, it may be tricky to distinguish the reflected signal from the proper one. Moreover, in the case of strong scattering, this misleading feature may not be discarded by putting an angle between the laser beam and the surface normal. This was tested by putting a large angle $\left(70^{\circ}\right)$ between the ceramic normal and the laser beam, and the two peaks remained with the same ratio. A method to determine the peak coming from the reflected laser beam in the case of strong scattering is discussed in Sec. V.

\section{B. Influence of the plasma density on the IVDF}

Previous measurements made in the same device showed an increase in the ion density at the sheath entrance before the characteristic density drop of the sheath ${ }^{19}$ which was not predicted by theoretical models. ${ }^{20,21}$ This rise of density is again observed, both for the metallic and ceramic plates even at low laser power (Fig. 8), and combines with the LIF saturation effect. The result is that the ratio between $\mathrm{P}^{+}$and $\mathrm{P}^{-}$evolves along the pre-sheath, and may go from values smaller than 1 to values larger than 1, as shown in Fig. 5. As the density rises along the pre-sheath, the number of metastable ions able to be excited by the laser rises, which causes the incident beam not to saturate the transition if the density becomes high enough. The

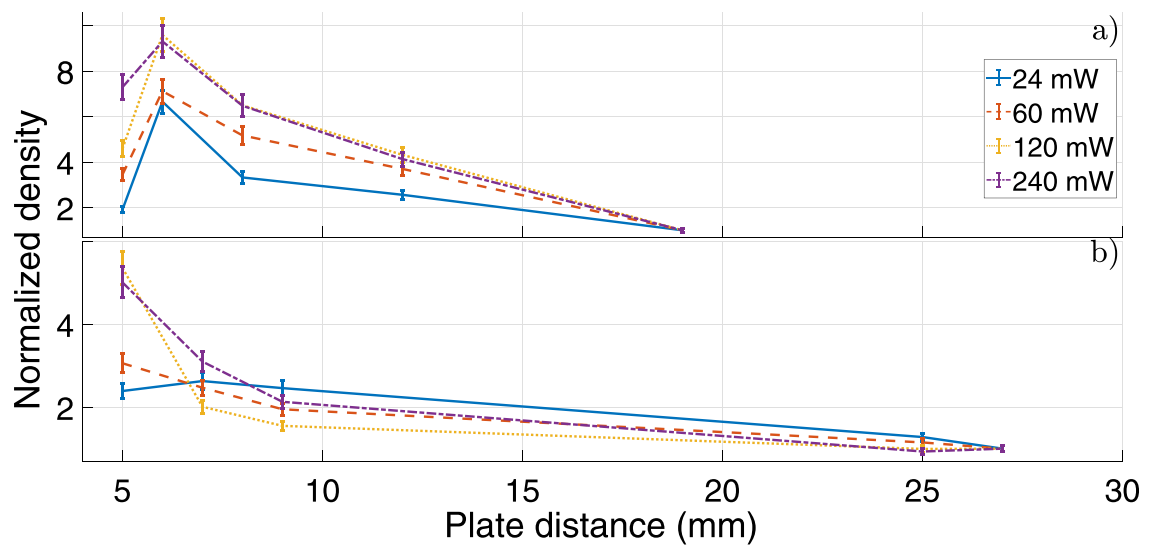

FIG. 8. Density of $P^{+}$in front of (a) the ceramic, and (b) the sample plate. The density is normalized to its value at the farthest location. The density drops for the ceramic but not for the metal plate at $5 \mathrm{~mm}$, although the fluid velocity is the same, suggesting that the ion flow in the pre-sheath/sheath is different between the two materials. 
explanation of the density rise being outside the scope of this paper, it will be given in another study.

\section{REFLECTED BEAM SIGNAL DEFORMATION DUE TO LASER BEAM SCATTERING}

In addition to the inversion of the ratio between $\mathrm{P}^{+}$and $\mathrm{P}^{-}$ maxima, the latter exhibits a strong asymmetry compared to the other, especially for the ceramic disk. This feature is due to the scattering of the reflected laser beam, and may be modeled as follows.

Let us consider the 3D IVDF, which can be split into a perpendicular and a parallel (with respect to the plate) contribution named $f_{\perp}$ and $f_{\|}$, such as $f_{i}=f_{\perp} f_{\|}$. These contributions read

$$
\begin{gathered}
\left.f_{\perp}\left(v_{z}\right)=A \exp \frac{-m_{i}\left(v_{z}-V\right)^{2}}{2 T_{i}}\right), \\
\left.f_{\|}\left(v_{x}, v_{y}\right)=B \exp \frac{-m_{i}\left(v_{x}^{2}+v_{y}^{2}\right)}{2 T_{i}}\right) .
\end{gathered}
$$

The IVDF is considered to be maxwellian for simplicity, and the perpendicular and parallel temperatures are chosen to be equal. As it is scattered at the plate surface, the reflected laser beam may excite ions of both contributions. This laser beam scattering is supposed to be ideal and follow Lambert's cosine law, which states that the intensity of a scattered beam on a surface is proportional to the cosine of the angle $\theta$ between the surface normal and the scattered beam propagation direction. ${ }^{37}$ The measured distribution function $f_{r 1}$ corresponds to the projection of the two IVDF components on the reflection cone of the scattered laser beam. The projection of an ion velocity $\mathbf{v}$ on a scattered laser photon of propagation direction $\mathbf{e}_{\gamma}$ is $v_{r}=\mathbf{v} \cdot \mathbf{e}_{\gamma}=v_{x} \cos \phi \sin \theta+v_{y} \sin \phi \sin \theta+v_{z} \cos \theta$, with $\phi$ the azimuthal angle. The projection of the IVDF on the reflected beam $f_{r 3}$ reads

$$
\begin{aligned}
f_{r 3}\left(v_{r}, \theta, \phi\right)= & \iiint \delta\left(v_{x} \cos \phi \sin \theta+v_{y} \sin \phi \sin \theta\right. \\
& \left.+v_{z} \cos \theta-v_{r}\right) f_{\perp} f_{\|} d^{3} \mathbf{v} .
\end{aligned}
$$

The measured distribution function $f_{r 1}$ is then given by

$$
f_{r 1}\left(v_{r}\right)=\int_{0}^{\pi / 2} \int_{0}^{2 \pi} f_{r 3}\left(v_{r}, \theta, \phi\right) \cos \theta \sin \theta d \theta d \phi .
$$

This multiple integral can be reduced to

$$
f_{r 1}\left(v_{r}\right)=\mathrm{AB} 4 \pi^{2} \int_{0}^{\mathrm{V}} \frac{1}{\mathrm{~V}^{2}} \exp \left(-\frac{\left(v_{r}-y\right)^{2}}{2}\right) y d y
$$

which is a definite integral

$$
\begin{aligned}
f_{r 1}\left(v_{r}\right)= & \frac{\mathrm{AB} 4 \pi^{2}}{\mathrm{~V}^{2}}\left(\sqrt{\frac{\pi}{2}} v_{r} \operatorname{erf}\left(\frac{\mathrm{V}-v_{r}}{\sqrt{2}}\right)+\sqrt{\frac{\pi}{2}} v_{r} \operatorname{erf}\left(\frac{v_{r}}{\sqrt{2}}\right)\right. \\
& \left.-e^{\left(\mathrm{V}-v_{r}\right)^{2} / 2}+e^{-v_{r}^{2} / 2}\right) .
\end{aligned}
$$

This function was used to fit the reflected beam part of the experimental IVDF. Concerning the incident beam signal, a fit with a maxwellian function appeared to be a rather good approximation. The results are shown in Fig. 9. The matching between the experimental data and the theoretical model is satisfactory (fitting parameters are given in Table I), except the discrepancy around $v=0$, which is discussed later on. On the other hand, the matching is quite poor for the metal plate reflected beam signal. This result confirms that the laser scattering at the ceramic surface is high, which
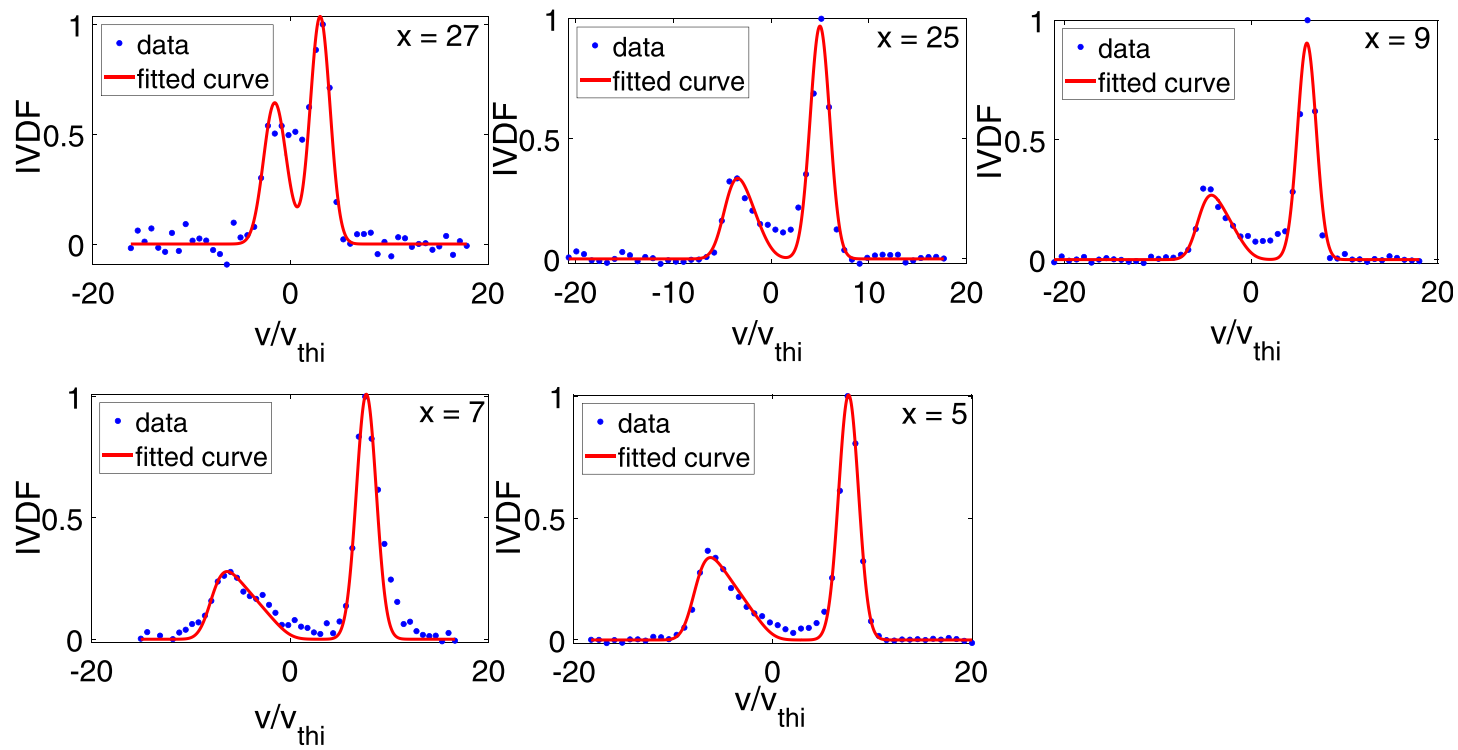

FIG. 9. Experimental IVDF, measured for $24 \mathrm{~mW}$ laser power, fitted by the diffuse reflection theoretical model. The IVDFs are normalized and the velocity is given in terms of $v_{\text {thi }}=\sqrt{T_{i} / m_{i}}$. Locations are given in $\mathrm{mm}$. 
TABLE I. Fitting parameters for the different IVDF shown in Fig. 9: ion temperature $T_{i}, A B$ coefficient in $f_{r 1}$, and drift velocity $V$. The dissymmetry between the two peaks most probable velocity $v_{\max }$ is due to the laser scattering.

\begin{tabular}{|c|c|c|c|c|c|c|}
\hline \multirow[b]{2}{*}{ Location (mm) } & \multirow[b]{2}{*}{$T_{i}(\mathrm{~K})$} & \multirow[b]{2}{*}{$A B$} & \multicolumn{2}{|c|}{$v_{\max }$} & \multicolumn{2}{|c|}{ V } \\
\hline & & & $P^{-}$ & $P^{+}$ & $P^{-}$ & $P^{+}$ \\
\hline 27 & 370 & 1.492 & -1.44 & 2.87 & -2.0 & 2.8 \\
\hline 25 & 260 & 1.008 & -3.4 & 5.0 & -4.7 & 5.0 \\
\hline 9 & 250 & 0.9057 & -4.3 & 5.9 & -5.6 & 5.9 \\
\hline 7 & 280 & 1.428 & -6.3 & 7.7 & -7.9 & 7.7 \\
\hline 5 & 370 & 1.188 & -6.3 & 7.6 & -7.8 & 7.6 \\
\hline
\end{tabular}

is coherent with the explanation provided in Sec. IV about the relative size of $\mathrm{P}^{+}$and $\mathrm{P}^{-}$.

The asymmetry of the reflected laser beam signal is due to the scattering. Also, this model provides an explanation on the observed difference between the drift velocities of both peaks. Indeed, as a non-negligible part of the ions are excited by photons propagating not perpendicularly with respect to their velocity, this shifts the measured drift velocity towards zero. The apparent most probable velocity and the fluid velocity computed from the model for each peak are shown in Table I. The model allows recovering the $\mathrm{P}^{-}$drift velocity that corresponds to the opposite of the $\mathrm{P}^{+}$drift velocity.

It also has to be noted that an additional component in the signal remains, around null velocities, which decreases as one gets closer to the plate. This contribution may be attributed to non-maxwellian IVDFs that are known to appear in the presheath. ${ }^{19,38}$ It could also be due to charge-exchange collisions, which drive the pre-sheath electric field.

The model was also tested for the IVDF measured in front of the metal disk and the matching appeared to be poor. The Lambertian scattering hypothesis is no more valid as the reflection is specular. Two shifted maxwellian VDFs give a consistent fit of the data.

This effect of the laser beam scattering may be used to discriminate the reflected beam signal from the incident one, especially if saturation is present and the first is larger than the second. When the perfect scattering approximation is not valid, for metal plates for instance, lowering the laser power remains a good way to avoid any bias due to the optical pumping saturation.

\section{CONCLUSIONS}

The LIF saturation effect due to high laser power on IVDF measurements in a plasma pre-sheath/sheath has been presented and discussed. For this study, a ceramic and a metallic plate embedded in a low temperature plasma of a multipolar device are used. Two main features have been highlighted.

The first one is the misleading increase in the LIF signal coming from the reflected laser beam, when the measurements are performed perpendicularly to the surface. If the laser power is too high, the incident beam may saturate the LIF transition, while the reflected one may not, especially if the reflection on the surface is diffuse. We also argue that the measurement volume, depending on the detection system resolution, has an influence on this phenomenon. The larger the volume, the higher the chance to observe this effect.

Countermeasures exist to unambiguously distinguish the proper LIF signal from the reflection artifact. An efficient way of identifying the reflected beam is to drill a hole in the sample and propagate the laser beam through it. However, this hole size should be of the order of the Debye length so that Debye screening is effective but it nevertheless induces a surface modification. This technique has been successfully applied in the present experiments to confirm the artifact nature of the $\mathrm{P}^{-}$peak (for the steel and ceramic plates). It is also possible to coat the surface with a light absorbent material, but it induces surface property modifications that may also change the sheath structure.

It is also shown that the reflected beam signal is asymmetric, which is due to the laser scattering. The scattered beam is able to excite ions which belong to the perpendicular VDF (with respect to the wall). This stretches the IVDF towards null velocities and shifts the maximum from its expected value, i.e., the opposite of the incident beam signal maximum.

The second feature is the influence of density gradients on the LIF measurement. When high laser power is used, the ion/ metastable ion density variations may be able to modify the ratio between the incident and reflected beam signals, which may complicate the interpretation of the data. This is true in the presheath, but could, most of the time, not be detected in the sheath. Indeed, regarding the high ion velocity in the sheath, and the typical wavelength scan range of the tunable lasers, one may just detect ions with velocity oriented toward the wall.

As a conclusion, we have highlighted that preliminary measurements should always be made in the bulk plasma to identify the laser power threshold associated with the LIF saturation effect. Low laser power associated with a large signal averaging is preferable to broadening deconvolution techniques in gradient regions, if the experiment duration remains reasonable.

\section{ACKNOWLEDGMENTS}

The authors would like to thank Yves Elskens for his precious help and advice about theoretical considerations.

\section{REFERENCES}

${ }^{\mathbf{1}}$ R. M. Measures, J. Appl. Phys. 39, 5232 (1968).

${ }^{2}$ R. A. Stern and J. A. Johnson, Phys. Rev. Lett. 34, 1548 (1975).

${ }^{3}$ D. N. Hill, S. Fornaca, and M. G. Wickham, Rev. Sci. Instrum. 54, 309 (1983).

${ }^{4}$ K. Dzierżega, K. Musiol, E. C. Benck, and J. R. Roberts, J. Appl. Phys. 80, 3196 (1996).

${ }^{5}$ F. M. Levinton and F. Trintchouk, Rev. Sci. Instrum. 72, 898 (2001).

${ }^{6}$ R. Hardin, X. Sun, and E. E. Scime, Rev. Sci. Instrum. 75, 4103 (2004).

${ }^{7}$ U. Czarnetzki, D. Luggenhöscher, and H. F. Döbele, Phys. Rev. Lett. 81, 4592 (1998)

${ }^{8}$ S. Mattingly and F. Skiff, Phys. Plasmas 25, 055707 (2018).

${ }^{9}$ J. Bowles, R. McWilliams, and N. Rynn, Phys. Plasmas 1, 3814 (1994)

${ }^{10}$ N. Claire, M. Dindelegan, G. Bachet, and F. Skiff, Rev. Sci. Instrum. 72, 4372 (2001).

${ }^{11}$ N. Claire, S. Mazouffre, C. Rebont, and F. Doveil, Phys. Plasmas 19, 032108 (2012).

${ }^{12}$ J. C. Camparo, Phys. Rev. A 39, 69 (1989).

${ }^{13}$ M. D. Bowden, Y. W. Choi, K. Muraoka, and M. Maeda, Appl. Phys. Lett. 66, 1059 (1995) 
${ }^{14}$ I. Romadanov, Y. Raitses, A. Diallo, K. Hara, I. D. Kaganovich, and A. Smolyakov, Phys. Plasmas 25, 033501 (2018).

${ }^{15}$ R. Altkorn and R. N. Zare, Ann. Rev. Phys. Chem. 35, 265 (1984).

${ }^{16}$ M. J. Goeckner and J. Goree, J. Vac. Sci. Technol. A 7, 977 (1989).

${ }^{17}$ M. J. Goeckner, J. Goreea, and T. E. Sheridan, Rev. Sci. Instrum. 64, 996 (1993).

${ }^{18}$ F. Chu and F. Skiff, Phys. Plasmas 25, 013506 (2018).

${ }^{19}$ N. Claire, G. Bachet, U. Stroth, and F. Doveil, Phys. Plasmas 13, 062103 (2006).

${ }^{20}$ S. Baalrud and C. Hegna, Plasma Sources Sci. Technol. 20, 025013 (2011).

${ }^{21}$ D. Coulette and G. Manfredi, Phys. Plasmas 22, 043505 (2015).

${ }^{22}$ R. Hood, B. Scheiner, S. Baalrud, M. Hopkins, E. Barnat, B. Yee, R. Merlino, and F. Skiff, Phys. Plasmas 23, 113503 (2016).

${ }^{23}$ E. V. Barnat and G. A. Hebner, J. Appl. Phys. 96, 4762 (2004).

${ }^{24}$ C.-S. Yip, N. Hershkowitz, G. Severn, and S. Baalrud, Phys. Plasmas 23, 050703 (2016).

${ }^{25}$ C.-S. Yip, N. Hershkowitz, and G. Severn, Phys. Rev. Lett. 104, 225003 (2010).

${ }^{26}$ L. Oksuz, M. A. Khedr, and N. Hershkowitz, Phys. Plasmas 8, 1729 (2001).

${ }^{27}$ L. Oksuz and N. Hershkowitz, Plasma Sources Sci. Technol. 14, 201 (2005).
${ }^{28}$ N. B. Moore, W. Gekelman, P. Pribyl, Y. Zhang, and M. J. Kushner, Phys. Plasmas 20, 083506 (2013)

${ }^{29}$ D. Lee, G. Severn, L. Oksuz, and N. Hershkowitz, J. Phys. D: Appl. Phys. 39 5230 (2006).

${ }^{30}$ T. Lunt, G. Fussmann, and O. Waldmann, Phys. Rev. Lett. 100, 175004 (2008).

${ }^{31}$ B. Jacobs, W. Gekelman, P. Pribyl, and M. Barnes, Phys. Rev. Lett. 105, 075001 (2010).

${ }^{32}$ M. K. Goeckner, J. Goree, and T. Sheridan, Phys. Fluids B: Plasma Phys. 4, 1663 (1992).

${ }^{33}$ M. Carrère, L. Chérigier, C. Arnas-Capeau, G. Bachet, and F. Doveil, Rev. Sci. Instrum. 67, 4124 (1996).

${ }^{34}$ E. Stamate, K. Inagaki, K. Ohe, and G. Popa, J. Phys. D: Appl. Phys. 32, 671 (1999).

${ }^{35}$ G. Hobbs and J. Wesson, Plasma Phys. 9, 85 (1967).

${ }^{36}$ M. D. Campanell, A. V. Khrabrov, and I. D. Kaganovich, Phys. Rev. Lett. 108, 235001 (2012).

${ }^{37}$ W. J. Smith, Modern Optical Engineering (SPIE Press, 2007).

${ }^{38}$ G. A. Emmert, R. M. Rieland, A. T. Mense, and J. N. Davidson, Phys. Fluids 23, 803 (1980). 